\title{
Inclusive jet production in two-photon collisions at LEP
}

\section{L3 Collaboration}

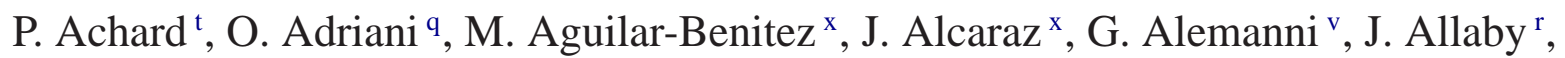
A. Aloisio ${ }^{\text {ab }}$, M.G. Alviggi ${ }^{\text {ab }}$, H. Anderhub ${ }^{\text {at }}$, V.P. Andreev ${ }^{\text {f,ag }}$, F. Anselmo ${ }^{\text {h }}$, A. Arefiev ${ }^{\text {aa }}$, T. Azemoon ${ }^{\text {c }}$, T. Aziz ${ }^{\text {i, P. Bagnaia }}{ }^{\text {al }}$, A. Bajo ${ }^{\mathrm{x}}$, G. Baksay ${ }^{\mathrm{y}}$, L. Baksay $^{\mathrm{y}}$, S.V. Baldew ${ }^{\text {b }}$, S. Banerjee ${ }^{\text {i }}$, Sw. Banerjee ${ }^{\mathrm{d}}$, A. Barczyk ${ }^{\text {at,ar }}$, R. Barillère ${ }^{\mathrm{r}}$, P. Bartalini ${ }^{\mathrm{v}}$, M. Basile ${ }^{\mathrm{h}}$, N. Batalova ${ }^{\mathrm{aq}}, \mathrm{R}$. Battiston ${ }^{\text {af }}$, A. Bay ${ }^{\mathrm{v}}$, F. Becattini ${ }^{\mathrm{q}}, \mathrm{U}$. Becker ${ }^{\mathrm{m}}$, F. Behner ${ }^{\text {at }}$, L. Bellucci ${ }^{q}$, R. Berbeco ${ }^{c}$, J. Berdugo ${ }^{\mathrm{x}}$, P. Berges ${ }^{\mathrm{m}}$, B. Bertucci ${ }^{\text {af }}$, B.L. Betev ${ }^{\text {at }}$, M. Biasini ${ }^{\text {af }}$, M. Biglietti ${ }^{\text {ab }}$, A. Biland ${ }^{\text {at }}$, J.J. Blaising ${ }^{\text {d }}$, S.C. Blyth ${ }^{\text {ah }}$, G.J. Bobbink ${ }^{\text {b }}$, A. Böhm ${ }^{\text {a }}$, L. Boldizsar ${ }^{1}$, B. Borgia ${ }^{\text {al }}$, S. Bottai ${ }^{\text {q }}$, D. Bourilkov ${ }^{\text {at }}$, M. Bourquin ${ }^{\mathrm{t}}$, S. Braccini ${ }^{\mathrm{t}}$, J.G. Branson ${ }^{\text {an }}$, F. Brochu ${ }^{\mathrm{d}}$, J.D. Burger ${ }^{\mathrm{m}}$, W.J. Burger ${ }^{\text {af }}$, X.D. Cai ${ }^{\mathrm{m}}$, M. Capell ${ }^{\mathrm{m}}$, G. Cara Romeo ${ }^{\mathrm{h}}$, G. Carlino ${ }^{\mathrm{ab}}$, A. Cartacci ${ }^{\mathrm{q}}$, J. Casaus ${ }^{\mathrm{x}}$, F. Cavallari al ${ }^{\text {a }}$ N. Cavallo ${ }^{\text {ai }}$, C. Cecchi ${ }^{\text {af }}$, M. Cerrada ${ }^{\mathrm{x}}$, M. Chamizo ${ }^{\mathrm{t}}$, Y.H. Chang ${ }^{\text {av }}$, M. Chemarin ${ }^{w}$, A. Chen ${ }^{\text {av }}$, G. Chen ${ }^{\mathrm{g}}$, G.M. Chen ${ }^{\mathrm{g}}$, H.F. Chen ${ }^{\mathrm{u}}$, H.S. Chen ${ }^{\mathrm{g}}$,

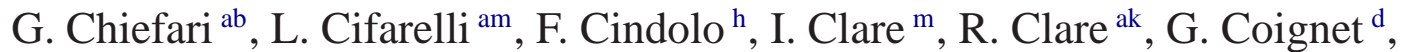
N. Colino ${ }^{x}$, S. Costantini ${ }^{\text {al }}$, B. de la Cruz ${ }^{\mathrm{x}}$, S. Cucciarelli af, J.A. van Dalen ${ }^{\text {ad }}$, R. de Asmundis ${ }^{\text {ab }}$, P. Déglon ${ }^{t}$, J. Debreczeni ${ }^{1}$, A. Degré ${ }^{\mathrm{d}}$, K. Dehmelt ${ }^{\mathrm{y}}$, K. Deiters ${ }^{\text {ar }}$, D. della Volpe ${ }^{\text {ab }}$, E. Delmeire ${ }^{t}$, P. Denes ${ }^{\text {aj }}$, F. DeNotaristefani ${ }^{\text {al }}$, A. De Salvo ${ }^{\text {at }}$, M. Diemoz ${ }^{\text {al }}$, M. Dierckxsens ${ }^{b}$, C. Dionisi ${ }^{\text {al }}$, M. Dittmar ${ }^{\text {at }}$, A. Doria ${ }^{\text {ab }}$, M.T. Dova $^{\mathrm{j}, 5}$, D. Duchesneau ${ }^{d}$, M. Duda ${ }^{\text {a }}$, B. Echenard ${ }^{\text {t }}$, A. Eline ${ }^{\text {r }}$, A. El Hage ${ }^{\text {a }}$, H. El Mamouni ${ }^{\text {w, }}$ A. Engler ${ }^{\text {ah }}$, F.J. Eppling ${ }^{\mathrm{m}}$, P. Extermann ${ }^{\mathrm{t}}$, M.A. Falagan ${ }^{\mathrm{x}}$, S. Falciano ${ }^{\text {al }}$, A. Favara ${ }^{\text {ae }}$, J. Fay ${ }^{\text {w }}$, O. Fedin ${ }^{\text {ag }}$, M. Felcini ${ }^{\text {at }}$, T. Ferguson ${ }^{\text {ah }}$, H. Fesefeldt ${ }^{\text {a }}$, E. Fiandrini ${ }^{\text {af }}$, J.H. Field ${ }^{\mathrm{t}}$, F. Filthaut ${ }^{\text {ad }}$, P.H. Fisher ${ }^{\mathrm{m}}$, W. Fisher ${ }^{\text {aj }}$, I. Fisk ${ }^{\text {an }}$, G. Forconi ${ }^{\mathrm{m}}$, K. Freudenreich ${ }^{\text {at }}$, C. Furetta ${ }^{\mathrm{z}}$, Yu. Galaktionov ${ }^{\mathrm{aa}, \mathrm{m}}$, S.N. Ganguli ${ }^{\mathrm{i}}$, P. Garcia-Abia ${ }^{\mathrm{x}}$, M. Gataullin ae, S. Gentile ${ }^{\text {al }}$, S. Giagu ${ }^{\text {al }}$, Z.F. Gong ${ }^{\text {u }}$, G. Grenier ${ }^{\text {w }}$, O. Grimm ${ }^{\text {at }}$,

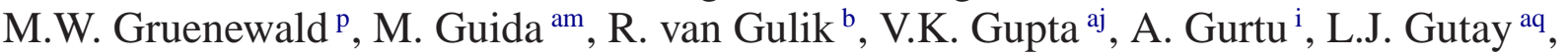
D. Haas ${ }^{\text {e }}$ D. Hatzifotiadou ${ }^{\text {h }}$, T. Hebbeker ${ }^{\mathrm{a}}$, A. Hervé ${ }^{\mathrm{r}}$, J. Hirschfelder ${ }^{\text {ah }}$, H. Hofer $^{\text {at }}$, M. Hohlmann ${ }^{y}$, G. Holzner ${ }^{\text {at }}$, S.R. Hou ${ }^{\text {av }}$, Y. Hu ${ }^{\text {ad }}$, B.N. Jin ${ }^{\mathrm{g}}$, L.W. Jones ${ }^{\mathrm{c}}$, P. de Jong ${ }^{b}$, I. Josa-Mutuberría ${ }^{x}$, D. Käfer ${ }^{a}$, M. Kaur ${ }^{\text {n }}$, M.N. Kienzle-Focacci ${ }^{\text {t, }}$ J.K. Kim ${ }^{\text {ap }}$, J. Kirkby ${ }^{\text {r }}$ W. Kittel ${ }^{\text {ad }}$, A. Klimentov ${ }^{\text {m,aa }}$, A.C. König ${ }^{\text {ad }}$, M. Kopal ${ }^{\text {aq }}$, 
V. Koutsenko ${ }^{\text {m,aa }}$, M. Kräber ${ }^{\text {at }}$, R.W. Kraemer ${ }^{\text {ah }}$, A. Krüger ${ }^{\text {as }}$, A. Kunin ${ }^{\text {m }}$, P. Ladron de Guevara ${ }^{\mathrm{x}}$, I. Laktineh ${ }^{\mathrm{w}}$, G. Landi ${ }^{\mathrm{q}}$, M. Lebeau ${ }^{\mathrm{r}}$, A. Lebedev ${ }^{\mathrm{m}}$, P. Lebrun ${ }^{\text {w }}$, P. Lecomte ${ }^{\text {at }}$, P. Lecoq ${ }^{\text {r }}$, P. Le Coultre ${ }^{\text {at }}$, J.M. Le Goff ${ }^{\text {r }}$, R. Leiste ${ }^{\text {as }}$, M. Levtchenko ${ }^{\text {z }}$, P. Levtchenko ${ }^{\text {ag }}$, C. Li ${ }^{\text {u }}$, S. Likhoded ${ }^{\text {as }}$, C.H. Lin ${ }^{\text {av }}$, W.T. Lin ${ }^{\text {av }}$, F.L. Linde ${ }^{\mathrm{b}}$, L. Lista $^{\mathrm{ab}}$, Z.A. Liu ${ }^{\mathrm{g}}$, W. Lohmann ${ }^{\text {as }}$, E. Longo ${ }^{\text {al }}$, Y.S. Lu ${ }^{\mathrm{g}}$, C. Luci ${ }^{\text {al }}$, L. Luminari ${ }^{\text {al }}$, W. Lustermann ${ }^{\text {at }}$, W.G. Ma ${ }^{\text {u }}$ L. Malgeri ${ }^{t}$, A. Malinin ${ }^{\text {aa }}$, C. Maña ${ }^{\mathrm{x}}$, J. Mans ${ }^{\text {aj }}$, J.P. Martin ${ }^{\text {w }}$, F. Marzano ${ }^{\text {al }}$, K. Mazumdar ${ }^{\mathrm{i}}$, R.R. McNeil ${ }^{\mathrm{f}}$, S. Mele ${ }^{\mathrm{r}, \mathrm{ab}}$, L. Merola ${ }^{\text {ab }}$, M. Meschini ${ }^{\mathrm{q}}$, W.J. Metzger ${ }^{\text {ad }}$, A. Mihul ${ }^{\text {k }}$, H. Milcent ${ }^{\mathrm{r}}$, G. Mirabelli ${ }^{\text {al }}$, J. Mnich ${ }^{\text {a }}$, G.B. Mohanty ${ }^{\mathrm{i}}$, G.S. Muanza ${ }^{\mathrm{w}}$, A.J.M. Muijs ${ }^{\mathrm{b}}$, B. Musicar ${ }^{\text {an }}$, M. Musy ${ }^{\text {al }}$, S. Nagy ${ }^{o}$, S. Natale ${ }^{\mathrm{t}}$, M. Napolitano ${ }^{\text {ab }}$, F. Nessi-Tedaldi ${ }^{\text {at }}$, H. Newman ${ }^{\text {ae }}$, A. Nisati ${ }^{\text {al }}$, T. Novak ${ }^{\text {ad }}$, H. Nowak ${ }^{\text {as }}$, R. Ofierzynski ${ }^{\text {at }}$, G. Organtini ${ }^{\text {al }}$, I. Pal ${ }^{\text {aq }}$, C. Palomares ${ }^{\mathrm{x}}$, P. Paolucci ${ }^{\text {ab }}$, R. Paramatti ${ }^{\text {al }}$, G. Passaleva ${ }^{\mathrm{q}}$, S. Patricelli ${ }^{\text {ab }}$, T. Paul ${ }^{\mathrm{j}}$, M. Pauluzzi $^{\text {af }}$, C. Paus ${ }^{\mathrm{m}}$, F. Pauss ${ }^{\text {at }}$, M. Pedace ${ }^{\text {al }}$, S. Pensotti ${ }^{\mathrm{z}}$, D. Perret-Gallix ${ }^{\mathrm{d}}$, B. Petersen ${ }^{\text {ad }}$, D. Piccolo ${ }^{\text {ab }}$, F. Pierella ${ }^{\text {h}}$, M. Pioppi ${ }^{\text {af }}$, P.A. Piroué ${ }^{\text {aj }}$, E. Pistolesi ${ }^{z}$, V. Plyaskin ${ }^{\text {aa }}$, M. Pohl ${ }^{\text {t }}$, V. Pojidaev ${ }^{\mathrm{q}}$, J. Pothier ${ }^{\mathrm{r}}$, D. Prokofiev ${ }^{\mathrm{ag}}$, J. Quartieri ${ }^{\text {am }}$, G. Rahal-Callot ${ }^{\text {at }}$, M.A. Rahaman ${ }^{i}$, P. Raics ${ }^{\circ}$, N. Raja ${ }^{i}$, R. Ramelli ${ }^{\text {at }}$, P.G. Rancoita ${ }^{\mathrm{z}}$, R. Ranieri ${ }^{\mathrm{q}}$, A. Raspereza ${ }^{\text {as }}$, P. Razis ${ }^{\text {ac }}$, D. Ren ${ }^{\text {at }}$, M. Rescigno ${ }^{\text {al }}$, S. Reucroft ${ }^{j}, S_{\text {S. Riemann }}{ }^{\text {as }}$, K. Riles ${ }^{\text {c }}$, B.P. Roe ${ }^{\text {c }}$, L. Romero ${ }^{x}$, A. Rosca ${ }^{\text {as }}$, C. Rosenbleck ${ }^{a}$, S. Rosier-Lees ${ }^{d}$, S. Roth ${ }^{\text {a }}$, J.A. Rubio ${ }^{\text {r }}$, G. Ruggiero ${ }^{\text {, }}$ H. Rykaczewski ${ }^{\text {at }}$, A. Sakharov ${ }^{\text {at }}$, S. Saremi $^{\text {f }}$, S. Sarkar al, J. Salicio ${ }^{r}$, E. Sanchez ${ }^{\text {x }}$, C. Schäfer ${ }^{r}$, V. Schegelsky ${ }^{\text {ag }}$, H. Schopper ${ }^{\text {au }}$, D.J. Schotanus ${ }^{\text {ad }}$, C. Sciacca ${ }^{\text {ab }}$, L. Servoli af, S. Shevchenko ae, N. Shivarov ${ }^{\text {ao }}$, V. Shoutko ${ }^{\mathrm{m}}$, E. Shumilov ${ }^{\text {aa }}$, A. Shvorob ${ }^{\text {ae }}$, D. Son ${ }^{\text {ap }}$, C. Souga ${ }^{\text {w }}$, P. Spillantini ${ }^{\text {, }}$, M. Steuer ${ }^{\mathrm{m}}$, D.P. Stickland ${ }^{\text {aj }}$, B. Stoyanov ${ }^{\text {ao }}$, A. Straessner ${ }^{\mathrm{t}}$, K. Sudhakar ${ }^{\mathrm{i}}$, G. Sultanov ${ }^{\text {ao }}$, L.Z. Sun ${ }^{\text {u }, ~ S . ~ S u s h k o v ~}{ }^{a}$, H. Suter ${ }^{\text {at }}$, J.D. Swain ${ }^{\mathrm{j}}$, Z. Szillasi ${ }^{\mathrm{y}, 3}$, X.W. Tang ${ }^{\mathrm{g}}$, P. Tarjan ${ }^{\mathrm{o}}$, L. Tauscher ${ }^{\mathrm{e}}$, L. Taylor ${ }^{\mathrm{j}}$, B. Tellili ${ }^{\mathrm{w}}$, D. Teyssier ${ }^{\mathrm{w}}$, C. Timmermans ${ }^{\text {ad }}$, S.C.C. Ting ${ }^{\mathrm{m}}$, S.M. Ting ${ }^{\mathrm{m}}$, S.C. Tonwar ${ }^{\mathrm{i}}$, J. Tóth ${ }^{1}$, C. Tully ${ }^{\text {aj, }}$ K.L. Tung ${ }^{g}$, J. Ulbricht ${ }^{\text {at }}$, E. Valente ${ }^{\text {al }}$, R.T. Van de Walle ${ }^{\text {ad }}$, R. Vasquez ${ }^{\text {aq }}$, V. Veszpremi ${ }^{y}$, G. Vesztergombi ${ }^{1}$, I. Vetlitsky $^{\text {aa }}$, D. Vicinanza ${ }^{\text {am }}$, G. Viertel ${ }^{\text {at }}$, S. Villa ${ }^{\text {ak }}$, M. Vivargent ${ }^{\mathrm{d}}, \mathrm{S}$. Vlachos ${ }^{\mathrm{e}}, \mathrm{I}_{\text {. Vodopianov }}{ }^{\mathrm{y}}, \mathrm{H}$. Vogel ${ }^{\text {ah }}, \mathrm{H}$. Vogt ${ }^{\text {as }}$,

I. Vorobiev ah,aa , A.A. Vorobyov ${ }^{\text {ag }}$, M. Wadhwa ${ }^{\mathrm{e}}$, Q. Wang ${ }^{\text {ad }}$, X.L. Wang ${ }^{\mathrm{u}}$, Z.M. Wang ${ }^{\text {u }}$, M. Weber ${ }^{\text {a }}$, P. Wienemann ${ }^{\text {a }}$, H. Wilkens ${ }^{\text {ad }}$, S. Wynhoff ${ }^{\text {aj }}$, L. Xia $^{\text {ae }}$, Z.Z. Xu ${ }^{\text {u }}$, J. Yamamoto ${ }^{c}$, B.Z. Yang ${ }^{\text {u }}$, C.G. Yang ${ }^{\mathrm{g}}$, H.J. Yang ${ }^{\mathrm{c}}$, M. Yang ${ }^{\mathrm{g}}$, S.C. Yeh ${ }^{\mathrm{aw}}$, An. Zalite ${ }^{\mathrm{ag}}$, Yu. Zalite ${ }^{\mathrm{ag}}$, Z.P. Zhang ${ }^{\mathrm{u}}$, J. Zhao ${ }^{\text {u }}$, G.Y. Zhu ${ }^{\mathrm{g}}$, R.Y. Zhu ${ }^{\text {ae }}$, H.L. Zhuang ${ }^{\mathrm{g}}$, A. Zichichi ${ }^{\mathrm{h}, \mathrm{r}, \mathrm{s}}$, B. Zimmermann ${ }^{\text {at }}$, M. Zöller ${ }^{\mathrm{a}}$

\footnotetext{
${ }^{a}$ III. Physikalisches Institut, RWTH, D-52056 Aachen, Germany ${ }^{1}$

${ }^{\mathrm{b}}$ National Institute for High Energy Physics, NIKHEF, and University of Amsterdam, NL-1009 DB Amsterdam, The Netherlands ${ }^{\mathrm{c}}$ University of Michigan, Ann Arbor, MI 48109, USA

d Laboratoire d'Annecy-le-Vieux de Physique des Particules, LAPP, IN2P3-CNRS, BP 110, F-74941 Annecy-le-Vieux cedex, France e Institute of Physics, University of Basel, CH-4056 Basel, Switzerland

${ }^{\mathrm{f}}$ Louisiana State University, Baton Rouge, LA 70803, USA
} 


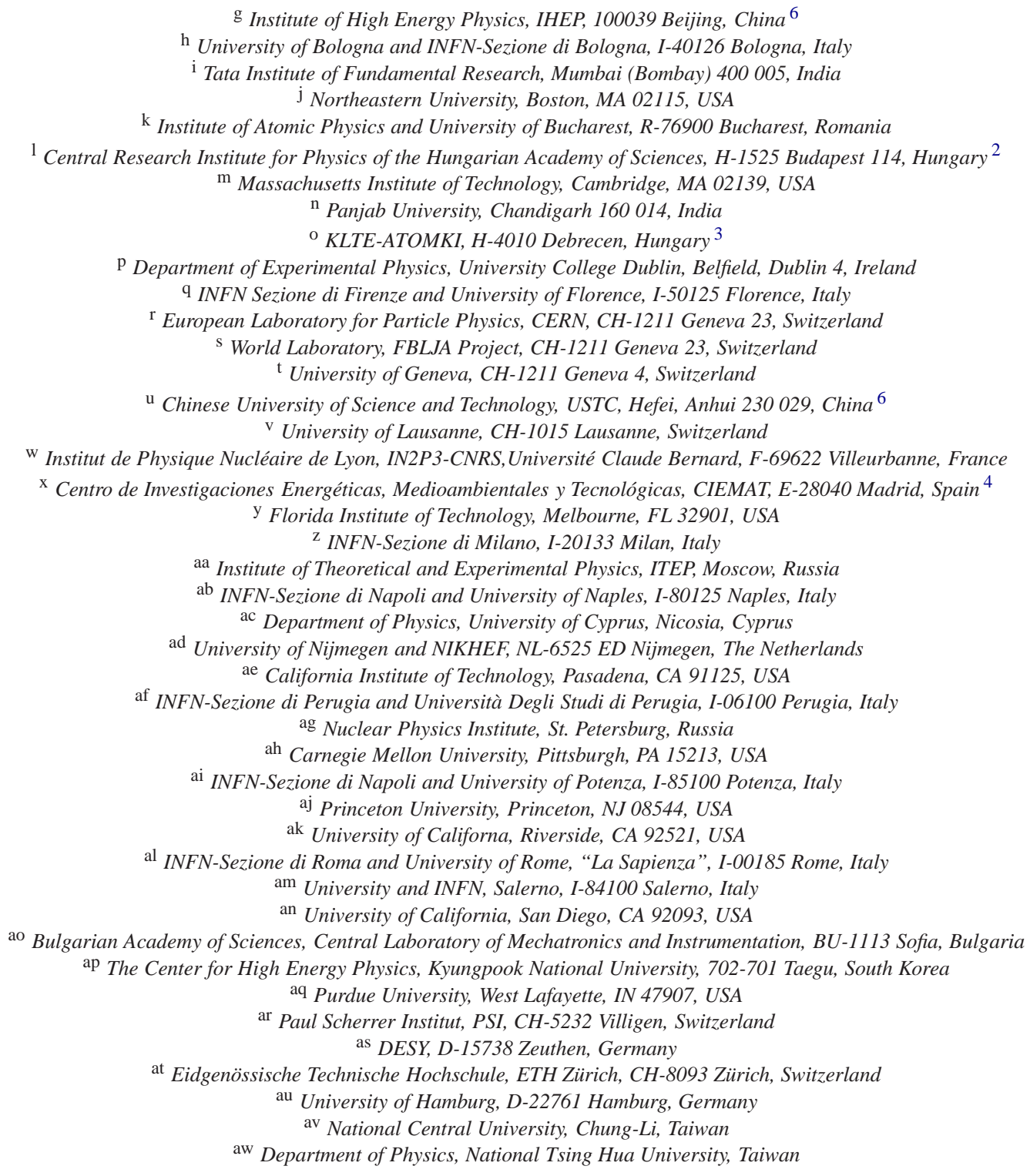

Received 27 August 2004; received in revised form 30 September 2004; accepted 1 October 2004

Available online 12 October 2004

Editor: L. Rolandi

\footnotetext{
${ }^{1}$ Supported by the German Bundesministerium für Bildung, Wissenschaft, Forschung und Technologie.

2 Supported by the Hungarian OTKA fund under contract numbers T019181, F023259 and T037350.

3 Also supported by the Hungarian OTKA fund under contract number T026178.

${ }^{4}$ Supported also by the Comisión Interministerial de Ciencia y Tecnología.

5 Also supported by CONICET and Universidad Nacional de La Plata, CC 67, 1900 La Plata, Argentina.

6 Supported by the National Natural Science Foundation of China.
} 


\begin{abstract}
Inclusive jet production, $\mathrm{e}^{+} \mathrm{e}^{-} \rightarrow \mathrm{e}^{+} \mathrm{e}^{-}$jet $\mathrm{X}$, is studied using $560 \mathrm{pb}^{-1}$ of data collected at LEP with the L3 detector at centre-of-mass energies between 189 and $209 \mathrm{GeV}$. The inclusive differential cross section is measured using a $k_{t}$ jet algorithm as a function of the jet transverse momentum, $p_{t}$, in the range $3<p_{t}<50 \mathrm{GeV}$ for a pseudorapidity, $\eta$, in the range $-1<\eta<1$. This cross section is well represented by a power law. For high $p_{t}$, the measured cross section is significantly higher than the NLO QCD predictions, as already observed for inclusive $\pi^{ \pm}$and $\pi^{0}$ production.
\end{abstract}

(C) 2004 Published by Elsevier B.V.

\section{Introduction}

Two-photon collisions are the main source of hadron production in the high-energy regime of LEP via the process $\mathrm{e}^{+} \mathrm{e}^{-} \rightarrow \mathrm{e}^{+} \mathrm{e}^{-} \gamma^{*} \gamma^{*} \rightarrow \mathrm{e}^{+} \mathrm{e}^{-}$hadrons. Hadrons with high transverse momentum are produced by the direct QED process $\gamma^{*} \gamma^{*} \rightarrow \mathrm{q} \overline{\mathrm{q}}$ or by QCD processes originating from the partonic content of the photon. Next-to-leading order (NLO) QCD calculations are available [1,2] for inclusive jet production in quasi-real two-photon interactions.

The L3 Collaboration published results on inclusive $\pi^{0}, \mathrm{~K}_{\mathrm{S}}^{0}$ [3] and charged hadron [4] production in quasi-real two-photon collisions. The inclusive $\pi^{0}$ and $\pi^{ \pm}$differential cross sections, measured as a function of transverse momentum, exhibit a clear excess over NLO QCD calculations [5] for large transverse momentum. In this Letter, inclusive jet production is studied, in similar two-photon interactions, for a centreof-mass energy of the two interacting photons, $W_{\gamma \gamma}$, greater than $5 \mathrm{GeV}$. The jets are measured in the transverse momentum range $3<p_{t}<50 \mathrm{GeV}$ and in the pseudo-rapidity interval $|\eta|<1$. The analysis of jet production allows a comparison of the measurements to NLO QCD predictions, expected to be largely independent of fragmentation functions and hadronisation models.

\section{Data and Monte Carlo}

The data used for this analysis were collected by the L3 detector [6] at centre-of-mass energies $\sqrt{s}=$ 189-209 GeV, with a luminosity weighted average value of $\sqrt{s}=198 \mathrm{GeV}$, and a total integrated luminosity of $560 \mathrm{pb}^{-1}$. Results on inclusive jet production at LEP for a smaller data sample at lower $\sqrt{s}$ were previously reported [7].
The process $\mathrm{e}^{+} \mathrm{e}^{-} \rightarrow \mathrm{e}^{+} \mathrm{e}^{-}$hadrons is modelled with the PYTHIA [8] event generator with an event sample two times larger than the data. In this generator, each photon can interact as a point-like particle (direct process), as a vector meson (VDM process) or as a resolved photon (resolved process), leading to six classes of events. Since both incoming photons are assumed to be on the mass shell, PYTHIA is modified to generate the photon flux in the equivalent photon approximation [9]. Predictions from the PHOJET [10] Monte Carlo program are also compared with the data. The following Monte Carlo generators are used to simulate the relevant background processes: KK2f [11] for $\mathrm{e}^{+} \mathrm{e}^{-} \rightarrow \mathrm{q} \overline{\mathrm{q}}(\gamma)$; KORALZ [12] for $\mathrm{e}^{+} \mathrm{e}^{-} \rightarrow$ $\tau^{+} \tau^{-}(\gamma)$; KORALW [13] for $\mathrm{e}^{+} \mathrm{e}^{-} \rightarrow \mathrm{W}^{+} \mathrm{W}^{-}$and DIAG36 [14] for $\mathrm{e}^{+} \mathrm{e}^{-} \rightarrow \mathrm{e}^{+} \mathrm{e}^{-} \tau^{+} \tau^{-}$. Jet hadronisation is simulated with the JETSET [8] parton shower algorithm. Events are simulated in the L3 detector using the GEANT [15] and GHEISHA [16] programs and passed through the same reconstruction program as the data. Time dependent detector inefficiencies, as monitored during each data taking period, are included in the simulations.

\section{Event selection}

Two-photon interaction events are collected predominantly by the track triggers [17] with a low transverse momentum threshold of about $150 \mathrm{MeV}$. The selection of $\mathrm{e}^{+} \mathrm{e}^{-} \rightarrow \mathrm{e}^{+} \mathrm{e}^{-}$hadrons events [18] consists of:

- A multiplicity cut. To select hadronic final states, at least six objects must be detected, where an object can be a track satisfying minimal quality requirements or a calorimetric cluster of energy greater than $100 \mathrm{MeV}$. 
- Energy cuts. To suppress background from beamgas and beam-wall interactions, the total energy in the electromagnetic calorimeter is required to be greater than $500 \mathrm{MeV}$. In order to exclude $\mathrm{e}^{+} \mathrm{e}^{-}$ annihilation events, the total energy deposited in the calorimeters must be less than $0.4 \sqrt{s}$.

- An anti-tag condition. Events with a cluster in the luminosity monitor, which covers the angular region $31<\theta<62 \mathrm{mrad}$, with an electromagnetic shower shape and energy greater than $30 \mathrm{GeV}$ are excluded.

- A mass cut. The mass of all the visible particles of the event, including clusters in the luminosity monitor, must be greater than $5 \mathrm{GeV}$. In this calculation, the pion mass is attributed to tracks and electromagnetic clusters are treated as massless. The visible mass distribution for data and Monte Carlo is shown, after all cuts, in Fig. 1. A wide range of masses is accessible.

About 3 million hadronic events are selected by these criteria. The background level of this sample is less than $1 \%$ and is mainly due to the $\mathrm{e}^{+} \mathrm{e}^{-} \rightarrow \mathrm{q} \overline{\mathrm{q}}(\gamma)$, $\mathrm{e}^{+} \mathrm{e}^{-} \rightarrow \tau^{+} \tau^{-}$and $\mathrm{e}^{+} \mathrm{e}^{-} \rightarrow \mathrm{e}^{+} \mathrm{e}^{-} \tau^{+} \tau^{-}$processes.

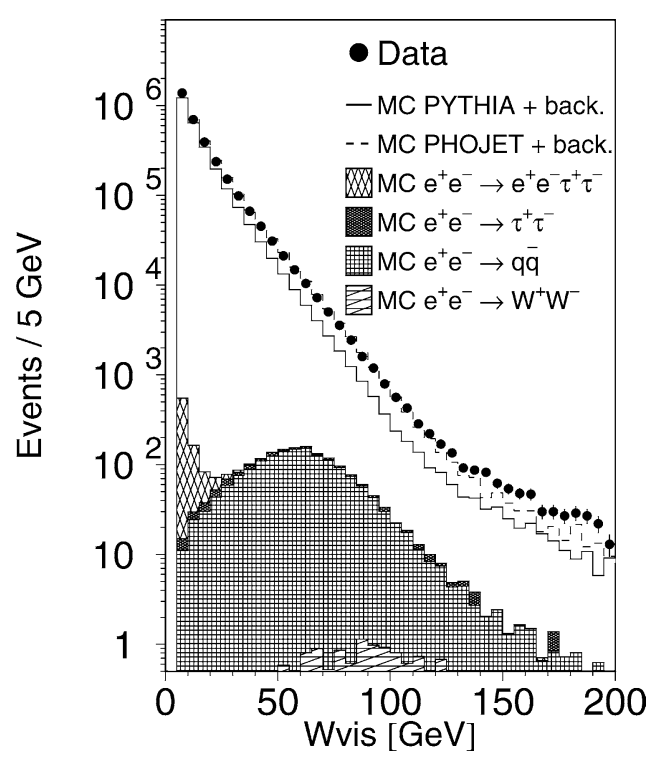

Fig. 1. Distribution of the visible mass for selected events. The Monte Carlo distributions are normalised to the luminosity of the data. Various contributions to the background (back) are shown as cumulative histograms.

\section{Jet definition and composition}

Jets are formed from good quality tracks and electromagnetic clusters. The tracks have a transverse momentum greater than $400 \mathrm{MeV}$, an absolute pseudorapidity less than 1 and a distance of closest approach to the primary vertex in the transverse plane less than $4 \mathrm{~mm}$. The number of hits must be greater than $80 \%$ of the maximum number expected from the track angle. For a transverse momentum less than $20 \mathrm{GeV}$, the momentum and direction of the tracks are measured with the central tracker. For the tracks with transverse momentum above $20 \mathrm{GeV}$, the track momenta are replaced with that derived from the energy of their associated cluster in the electromagnetic and hadronic calorimeters, assuming the pion mass. Tracks associated with muon chamber hits are rejected. An electromagnetic cluster must have an energy greater than $100 \mathrm{MeV}$ in at least 2 neighbouring BGO crystals and an absolute pseudorapidity less than 3.4. There should be no charged track within an angle of $200 \mathrm{mrad}$ around the cluster direction and the associated energy in the hadron calorimeter must be less than $20 \%$ of the electromagnetic energy.

Jets are constructed using the $k_{t}$ jet algorithm KTCLUS [19]. This algorithm uses cylindrical geometry in which the distance between two objects $i, j$ of transverse momenta $p_{t i}$ and $p_{t j}$ is defined as $d_{i j}=$ $\min \left(p_{t i}^{2}, p_{t j}^{2}\right)\left[\left(\eta_{i}-\eta_{j}\right)^{2}+\left(\Phi_{i}-\Phi_{j}\right)^{2}\right] / D^{2}$ where $\eta_{i}$ and $\eta_{j}$ are the pseudorapidities of the objects, $\Phi_{i}$ and $\Phi_{j}$ their azimuthal angles with respect to the beam axis and $D$ is a parameter of the algorithm which determines the size of the jet. The standard value $D=1$ is used. A distance parameter $d_{k}$ equal to $p_{t k}^{2}$ is also associated to each object. At the first iteration of the algorithm, the objects are the tracks and electromagnetic clusters defined above. At each iteration of the algorithm, the $d_{i j}$ and $d_{k}$ are ordered. If the smallest distance is a $d_{i j}$, the corresponding objects $i$ and $j$ are replaced by a new object, a "precluster", formed by adding the 4-momenta of the objects $i$ and $j$. If the smallest distance is a $d_{k}$ associated with a particle, this is considered as a "beam jet" particle and is removed from the list of objects. If the smallest distance is a $d_{k}$ associated with a precluster, this defines a "hard jet" and is removed from the list of objects. The procedure is iterated until all objects define beam or hard 
Table 1

Mean value and standard deviation (in brackets) of multiplicities and $p_{t}$ fractions for the jets in data and Monte Carlo events, at generator level as well as after reconstruction. The uncertainties on the mean values are quoted for the data. For Monte Carlo, they are always lower than the precision of the last digit

\begin{tabular}{|c|c|c|c|c|c|c|}
\hline \multirow{2}{*}{\multicolumn{2}{|c|}{ Variable }} & \multirow[t]{2}{*}{ Data } & \multicolumn{2}{|l|}{ PYTHIA } & \multicolumn{2}{|l|}{ PHOJET } \\
\hline & & & Reconstructed & Generated & Reconstructed & Generated \\
\hline \multirow{2}{*}{\multicolumn{2}{|c|}{$\begin{array}{l}\text { Total number of jets } \\
\text { Number of jets/event }\end{array}$}} & 68792 & 107140 & 188302 & 65781 & 105633 \\
\hline & & $1.2 \pm 0.1(0.5)$ & $1.4(0.7)$ & $1.3(0.7)$ & $1.2(0.4)$ & $1.1(0.5)$ \\
\hline \multicolumn{2}{|l|}{$N($ particles $) /$ jet } & $6.1 \pm 0.1(2.5)$ & $5.4(2.3)$ & $5.3(2.4)$ & $5.7(2.4)$ & $6.1(2.4)$ \\
\hline \multicolumn{2}{|c|}{$N$ (particles) outside jets } & $14.4 \pm 0.1(8.4)$ & $10.0(7.0)$ & $13.6(9.3)$ & $12.4(7.3)$ & $18.4(8.8)$ \\
\hline \multirow[t]{4}{*}{$N($ tracks $) /$ jet } & $3<p_{t}<5 \mathrm{GeV}$ & $2.2 \pm 0.1(1.3)$ & $2.3(1.3)$ & & $2.4(1.3)$ & \\
\hline & $5<p_{t}<10 \mathrm{GeV}$ & $2.4 \pm 0.1(1.3)$ & $2.6(1.3)$ & & $2.8(1.4)$ & \\
\hline & $10<p_{t}<25 \mathrm{GeV}$ & $2.5 \pm 0.1(1.6)$ & $2.9(1.3)$ & & $3.0(1.6)$ & \\
\hline & $25<p_{t}<45 \mathrm{GeV}$ & $2.7 \pm 0.2(1.7)$ & $3.3(1.6)$ & & - & \\
\hline \multirow[t]{4}{*}{$N($ clusters $) /$ jet } & $3<p_{t}<5 \mathrm{GeV}$ & $3.7 \pm 0.1(2.4)$ & $2.0(2.0)$ & & $3.1(2.2)$ & \\
\hline & $5<p_{t}<10 \mathrm{GeV}$ & $3.9 \pm 0.1(2.6)$ & $1.8(1.9)$ & & $3.3(2.4)$ & \\
\hline & $10<p_{t}<25 \mathrm{GeV}$ & $3.9 \pm 0.1(3.0)$ & $1.6(1.8)$ & & $3.3(2.5)$ & \\
\hline & $25<p_{t}<45 \mathrm{GeV}$ & $3.8 \pm 0.3(3.0)$ & $1.4(1.7)$ & & - & \\
\hline \multirow[t]{4}{*}{$p_{t}$ (leading $) / p_{t}$} & $3<p_{t}<5 \mathrm{GeV}$ & $0.50 \pm 0.01(0.18)$ & $0.53(0.18)$ & $0.50(0.18)$ & $0.51(0.18)$ & $0.46(0.17)$ \\
\hline & $5<p_{t}<10 \mathrm{GeV}$ & $0.54 \pm 0.01(0.20)$ & $0.55(0.19)$ & $0.50(0.20)$ & $0.52(0.19)$ & $0.43(0.17)$ \\
\hline & $10<p_{t}<25 \mathrm{GeV}$ & $0.63 \pm 0.01(0.23)$ & $0.60(0.20)$ & $0.48(0.22)$ & $0.60(0.24)$ & $0.39(0.19)$ \\
\hline & $25<p_{t}<45 \mathrm{GeV}$ & $0.69 \pm 0.03(0.23)$ & $0.56(0.14)$ & $0.47(0.25)$ & - & - \\
\hline
\end{tabular}

jets. Only hard jets with $p_{t}>3 \mathrm{GeV}$ and $|\eta|<1$ are further considered for this analysis.

In Table 1, the data are compared to the Monte Carlo at reconstructed and generated levels for: the number of jets, the mean number of jets per event with at least one jet, the mean number of particles per jet and outside the jets. For different $p_{t}$ intervals, comparisons are made of the mean number of tracks and electromagnetic clusters per jet and of transverse momentum of the leading particle divided by that of the jet. The standard deviations of these distributions are also quoted. For Monte Carlo at generator level, all particles with mean life time less than $3 \times 10^{-10} \mathrm{~s}$ are allowed to decay and jets are formed from the photons, charged pions, charged and neutral kaons, protons and neutrons. Both Monte Carlo programs underestimate the number of particles inside and outside the jets. The predicted number of electromagnetic clusters is too low for all $p_{t}$. The amount of energy carried by the most energetic particle of the jet is correctly reproduced, except in the highest $p_{t}$ interval. The number of particles per jet is shown in Fig. 2 .

Fig. 3 shows the distributions of $|\eta|$ for particles, i.e., clusters and tracks, tracks and jets in two inter-

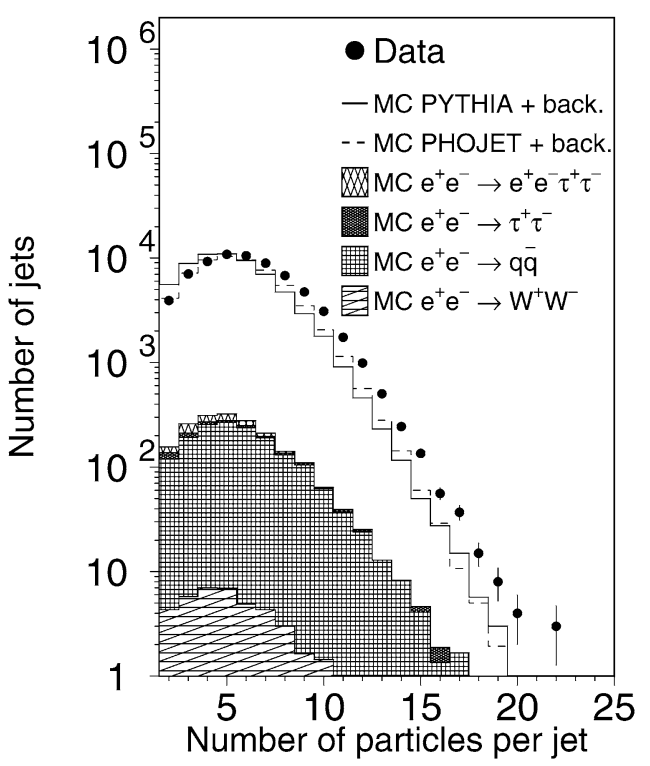

Fig. 2. Distribution of the number of particles per jet for jets with $p_{t}>3 \mathrm{GeV}$ and $|\eta|<1$. The Monte Carlo distributions are normalised to the luminosity of the data. Various contributions to the background (back) are shown as cumulative histograms. 

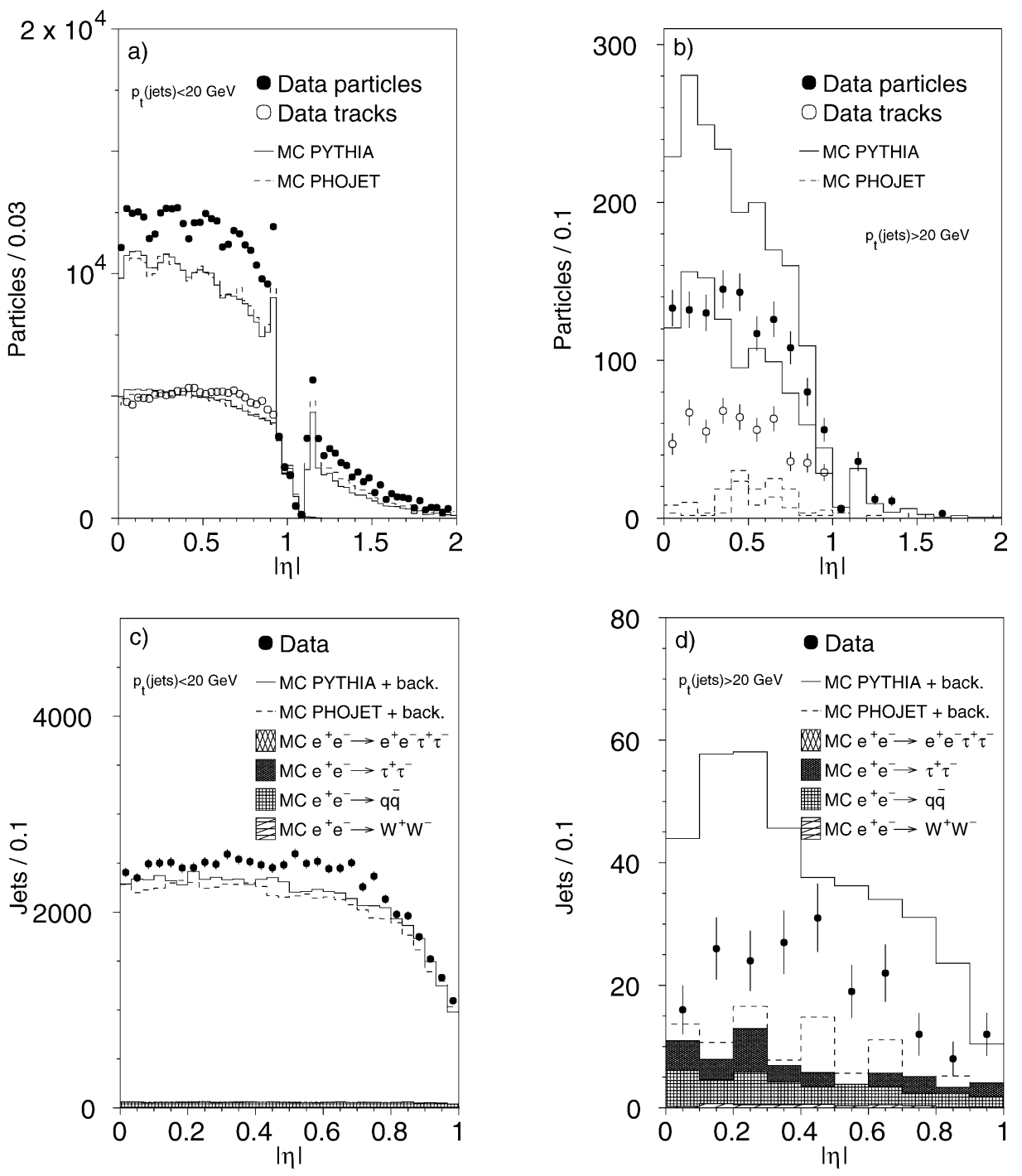

Fig. 3. Distributions of the pseudo rapidity $|\eta|$ for (a) and (b) particles and tracks used to form jets with $p_{t}<20 \mathrm{GeV}$ and $p_{t} \geqslant 20 \mathrm{GeV}$, respectively. "Particles" include both calorimetric clusters and tracks. (c) and (d) distributions of $|\eta|$ for reconstructed jets with $p_{t}<20 \mathrm{GeV}$ and $p_{t} \geqslant 20 \mathrm{GeV}$, respectively. The Monte Carlo distributions are normalised to the luminosity of the data. In (a) and (b) the higher Monte Carlo lines refer to particles and the lower ones to tracks. Various contributions to the background are shown as cumulative histograms in (c) and (d).

vals of the jet transverse momentum, $p_{t}<20 \mathrm{GeV}$ and $p_{t} \geqslant 20 \mathrm{GeV}$. The detector acceptance for tracks, calorimetric clusters and jets is well reproduced by Monte Carlo models.

\section{Differential cross section}

The differential cross section for inclusive jet production as a function of $p_{t}$ is measured for $W_{\gamma \gamma} \geqslant$
$5 \mathrm{GeV}$, with a mean value of $\left\langle W_{\gamma \gamma}\right\rangle \simeq 30 \mathrm{GeV}$, and a photon virtuality $Q^{2}<8 \mathrm{GeV}^{2}$, with $\left\langle Q^{2}\right\rangle \simeq$ $0.2 \mathrm{GeV}^{2}$. This phase space is defined by Monte Carlo generator-level cuts. Results are presented in $9 p_{t}$ bins between 3 and $50 \mathrm{GeV}$.

The $p_{t}$ distribution of the jets is presented in Fig. 4 . The total background is listed in Table 2. Events from the $\mathrm{e}^{+} \mathrm{e}^{-} \rightarrow \mathrm{e}^{+} \mathrm{e}^{-} \tau^{+} \tau^{-}$process dominate the background at low $p_{t}$ while hadronic and tau-pair annihi- 
lation events dominate it at high $p_{t}$. To measure the cross section, the background is subtracted bin-by-bin. The migration due to the $p_{t}$ resolution is corrected by a one-step Bayesian unfolding [20]. The data are corrected for the selection efficiency which includes acceptance, and is calculated bin-by-bin as the ratio of the number of fully simulated jets selected in PYTHIA over the number of generated jets, as formed by the KTCLUS algorithm applied to particles at genera-

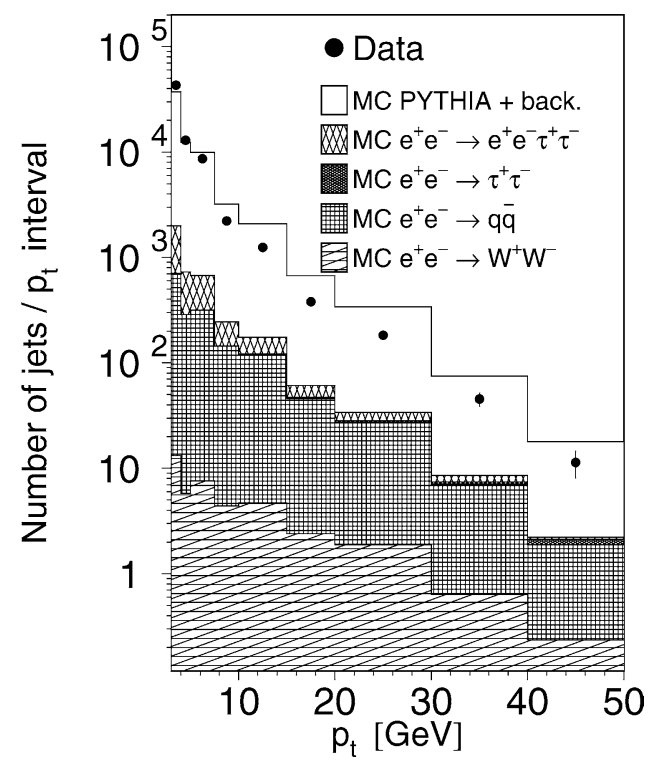

Fig. 4. Distribution of the number of jets with $|\eta|<1$ as a function of $p_{t}$. The Monte Carlo distributions are normalised to the luminosity of the data. Various contributions to the background (back) are shown as cumulative histograms. tor level. The efficiency decreases with $p_{t}$ from $61 \%$ to $15 \%$.

The level 1 trigger efficiency is obtained by comparing the number of events accepted by the independent track and calorimetric energy triggers [21]. It varies from $97 \%$ to $100 \%$. The efficiency of higher level triggers is about $98 \%$ and is measured using prescaled events. The differential cross section and the overall efficiency, which take into account selection and trigger efficiencies, are given as a function of $p_{t}$ in Table 2.

Sources of systematic uncertainties on the cross section measurements are the uncertainties on the estimation of the selection and trigger efficiencies, the limited Monte Carlo statistics, the background subtraction procedure, the selection procedure and the Monte Carlo modelling. Their contributions are shown in Table 3. The uncertainty due to the selection procedure is evaluated by repeating the analysis with different selection criteria: the multiplicity cut is moved to 5 and to 7 objects, the requirement on the number of hits of the tracks is moved to $70 \%$ of those expected, the isolation angle of clusters is moved to $100 \mathrm{mrad}$, and jets with a particle accounting for more than $90 \%$ of the jet transverse momentum are rejected. The sum in quadrature of the differences between these and the reference results is assigned as systematic uncertainty in Table 3. Varying other criteria, such as the energy cut, the minimum cluster energy or the threshold where the track energy is defined by calorimeters, gives negligible contributions. To evaluate the uncertainty on the Monte Carlo modelling, the selection efficiency is determined using only one of the PYTHIA

Table 2

Background level, reconstruction efficiency, trigger efficiency and differential cross section as a function of $p_{t}$ for $|\eta|<1$ and $W_{\gamma \gamma}>5 \mathrm{GeV}$. The first uncertainty is statistical and the second systematic. The average value of $p_{t}$ for each bin, $\left\langle p_{t}\right\rangle$, is also given

\begin{tabular}{lcclll}
\hline $\begin{array}{l}p_{t} \\
{[\mathrm{GeV}]}\end{array}$ & $\begin{array}{l}\left\langle p_{t}\right\rangle \\
{[\mathrm{GeV}]}\end{array}$ & $\begin{array}{l}\text { Background } \\
{[\%]}\end{array}$ & $\begin{array}{l}\text { Reconstruction } \\
\text { efficiency [\%] }\end{array}$ & $\begin{array}{l}\text { Trigger } \\
\text { efficiency [\%] }\end{array}$ & $\begin{array}{l}d \sigma / d p_{t} \\
{[\mathrm{pb} / \mathrm{GeV}]}\end{array}$ \\
\hline $3-4$ & 3.4 & $4.6 \pm 0.1$ & $60.8 \pm 0.2$ & $95.8 \pm 0.3$ & $(13 \pm 1 \pm 1) \times 10^{1}$ \\
$4-5$ & 4.4 & $5.6 \pm 0.1$ & $57.2 \pm 0.3$ & $95.9 \pm 0.5$ & $(40 \pm 1 \pm 3)$ \\
$5-7.5$ & 5.9 & $7.8 \pm 0.1$ & $53.2 \pm 0.3$ & $96.2 \pm 0.5$ & $(11 \pm 1 \pm 1)$ \\
$7.5-10$ & 8.5 & $11.1 \pm 0.1$ & $48.9 \pm 0.5$ & $96.6 \pm 1.0$ & $(30 \pm 1 \pm 2) \times 10^{-1}$ \\
$10-15$ & 11.9 & $14.0 \pm 0.2$ & $44.9 \pm 0.6$ & $96.8 \pm 1.4$ & $(88 \pm 3 \pm 7) \times 10^{-2}$ \\
$15-20$ & 17.1 & $16.0 \pm 0.4$ & $39.2 \pm 0.9$ & $96.9 \pm 2.0$ & $(30 \pm 2 \pm 3) \times 10^{-2}$ \\
$20-30$ & 24.0 & $18.6 \pm 0.8$ & $31.6 \pm 0.8$ & $97.3 \pm 2.1$ & $(90 \pm 7 \pm 8) \times 10^{-3}$ \\
$30-40$ & 34.1 & $18.9 \pm 1.5$ & $20.5 \pm 1.3$ & $97.3 \pm 2.5$ & $(31 \pm 5 \pm 2) \times 10^{-3}$ \\
$40-50$ & 44.7 & $19.6 \pm 1.6$ & $15.2 \pm 1.9$ & $98.5 \pm 2.8$ & $(11 \pm 3 \pm 2) \times 10^{-3}$ \\
\hline
\end{tabular}


Table 3

Systematic uncertainties on the inclusive jet cross section as a function of $p_{t}$

\begin{tabular}{llllcc}
\hline $\begin{array}{l}p_{t} \\
{[\mathrm{GeV}]}\end{array}$ & $\begin{array}{l}\text { Trigger } \\
\text { efficiency [\%] }\end{array}$ & $\begin{array}{l}\text { Monte Carlo } \\
\text { statistics [\%] }\end{array}$ & $\begin{array}{l}\text { Background } \\
\text { subtraction [\%] }\end{array}$ & $\begin{array}{l}\text { Selection } \\
\text { procedure [\%] }\end{array}$ & $\begin{array}{l}\text { Monte Carlo } \\
\text { modelling [\%] }\end{array}$ \\
\hline $3-4$ & 0.3 & 0.3 & $<0.1$ & 8.4 & 0.3 \\
$4-5$ & 0.5 & 0.5 & 0.2 & 7.0 & 1.3 \\
$5-7.5$ & 0.5 & 0.5 & 0.3 & 6.6 & 1.5 \\
$7.5-10$ & 1.0 & 1.0 & 0.6 & 4.8 & 2.4 \\
$10-15$ & 1.4 & 1.3 & 0.9 & 7.0 & 3.0 \\
$15-20$ & 2.1 & 2.4 & 1.7 & 6.0 & 3.3 \\
$20-30$ & 2.2 & 2.6 & 2.7 & $<0.1$ & 6.3 \\
$30-40$ & 2.6 & 6.4 & 5.2 & $<0.1$ & 12.4 \\
$40-50$ & 2.8 & 12.4 & 9.6 & & \\
\hline
\end{tabular}
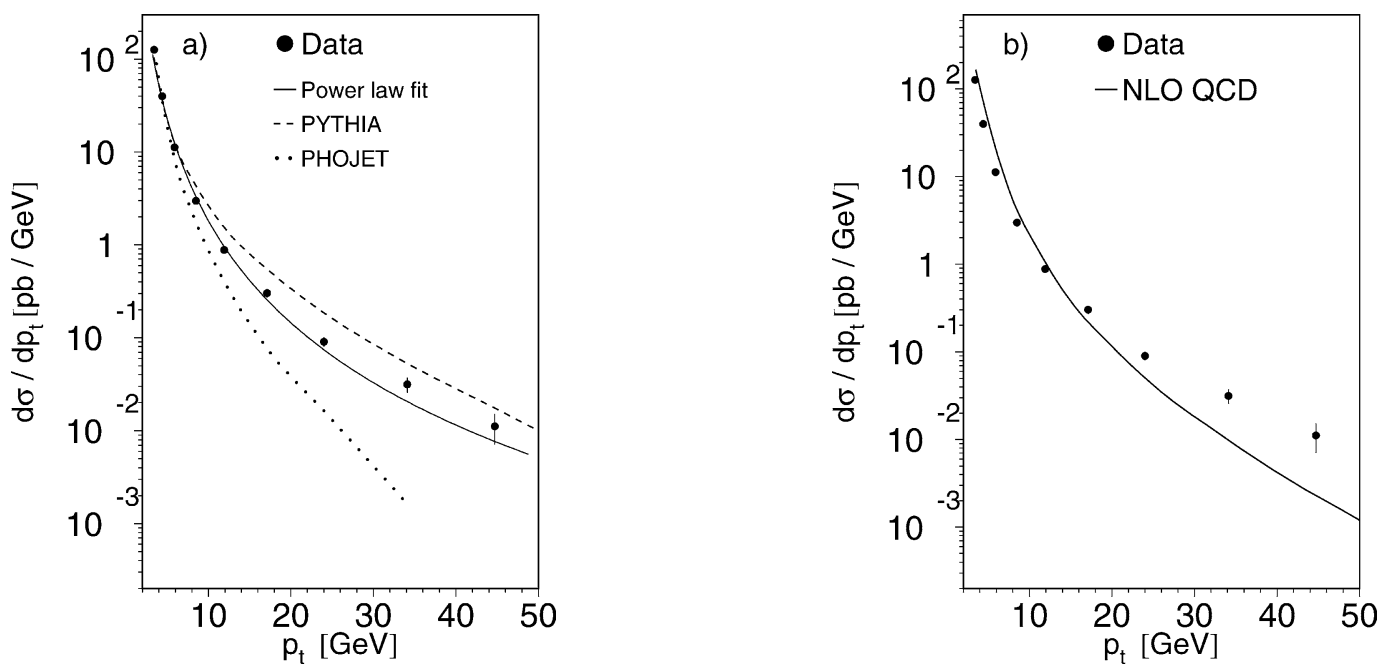

Fig. 5. Inclusive jet differential cross section $d \sigma / d p_{t}$ (a) compared to PYTHIA and PHOJET Monte Carlo predictions and the result of a power law fit (solid line); (b) compared to NLO QCD calculations [2] (solid line). The theoretical scale uncertainty is less than $20 \%$.

subprocesses: VDM-VDM, direct-direct or resolvedresolved. The systematic uncertainty is assigned as the maximum difference between these values and the reference Monte Carlo.

The differential cross sections as a function of $|\eta|$ are uniform within the experimental uncertainties for both $p_{t}<20 \mathrm{GeV}$ and $p_{t}>20 \mathrm{GeV}$, albeit in the latter case these uncertainties are large.

The differential cross section $d \sigma / d p_{t}$ is described by a power law function $A p_{t}^{-B}$, as expected from the onset of hard QCD processes, with $B=3.65 \pm 0.07$. The result of the fit is shown in Fig. 5(a) together with a comparison to Monte Carlo predictions.

In Fig. 5(b) the data are also compared to analytical NLO QCD predictions [2]. For this calculation, the flux of quasi-real photons is obtained using the improved Weizsäcker-Williams formula [22]. The interacting particles can be point-like photons or partons from the $\gamma \rightarrow \mathrm{q} \overline{\mathrm{q}}$ process, which evolve into quarks and gluons. The GRV-HO parton density functions of Ref. [23] are used and all elementary $2 \rightarrow 2$ and $2 \rightarrow 3$ processes are considered. The parameter $\Lambda^{(5)}$ is set to $130 \mathrm{MeV}$. The renormalization and factorisation scales are taken to be equal: $\mu=M=E_{t} / 2$ [1]. To assign uncertainties, the scale is varied by a factor $1 / 2$ or 2 , which gives a change in the prediction less than $20 \%$. The results of this calculation agree [2] with those described in Ref. [24]. An additional uncertainty in comparison with NLO QCD, which is not considered here, might arise from the modeling of the hadronisa- 


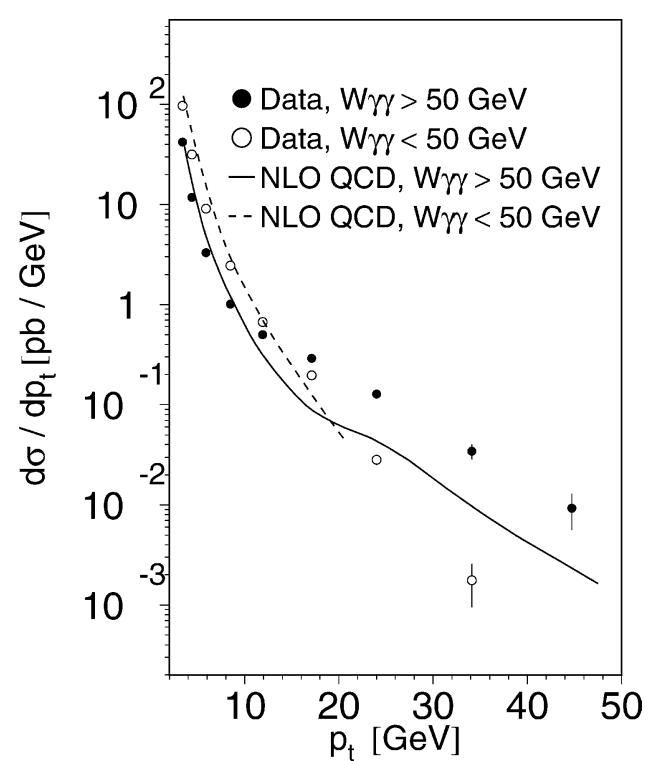

Fig. 6. Inclusive jet differential cross section $d \sigma / d p_{t}$ for events with two-photon centre-of-mass energy, $W_{\gamma \gamma}$, below and above $50 \mathrm{GeV}$. NLO QCD calculations [2] are superimposed to the data. The discontinuity around $25 \mathrm{GeV}$ is due to the direct contribution.

tion process. In a similar study [25] it was evaluated to be below $10 \%$ for $p_{t}>10 \mathrm{GeV}$ and decreasing with increasing $p_{t}$. The agreement with the data is poor in the high- $p_{t}$ range, as previously observed in the case of inclusive $\pi^{0}$ [3] and $\pi^{ \pm}$[4] production in similar two-photon reactions. In Fig. 6, the data are divided in two $W_{\gamma \gamma}$ ranges, $W_{\gamma \gamma}>50 \mathrm{GeV}$ and $W_{\gamma \gamma} \geqslant 50 \mathrm{GeV}$ and compared to the analytical NLO QCD predictions [2]. For $W_{\gamma \gamma} \geqslant 50 \mathrm{GeV}$ there is a clear discontinuity in the slope near $p_{t}=25 \mathrm{GeV}$, due to the direct contribution. At high $p_{t}$, the disagreement between data and theoretical calculations is still present.

\section{References}

[1] S. Frixione, Nucl. Phys. B 507 (1997) 295; S. Frixione, G. Ridolfi, Nucl. Phys. B 507 (1997) 315.

[2] L. Bertora, Nucl. Phys. B (Proc. Suppl.) 126 (2004) 134; S. Frixione, L. Bertora, private communication. We thank the authors for providing us with the NLO QCD calculations and for useful discussions.
[3] L3 Collaboration, P. Achard, et al., Phys. Lett. B 524 (2002) 44.

[4] L3 Collaboration, P. Achard, et al., Phys. Lett. B 554 (2003) 105.

[5] J. Binnewies, B.A. Kniehl, G. Kramer, Phys. Rev. D 53 (1996) 6110.

[6] L3 Collaboration, B. Adeva, et al., Nucl. Instrum. Methods A 289 (1990) 35;

M. Chemarin, et al., Nucl. Instrum. Methods A 349 (1994) 345; M. Acciarri, et al., Nucl. Instrum. Methods A 351 (1994) 300; G. Basti, et al., Nucl. Instrum. Methods A 374 (1996) 293; I.C. Brock, et al., Nucl. Instrum. Methods A 381 (1996) 236; A. Adam, et al., Nucl. Instrum. Methods A 383 (1996) 342.

[7] OPAL Collaboration, K. Ackerstaff, et al., Z. Phys. C 73 (1997) 433.

[8] PYTHIA version 5.722 and JETSET version 7.409 are used with default options:

T. Sjöstrand, Comput. Phys. Commun. 82 (1994) 74.

[9] V.M. Budnev, et al., Phys. Rep. 15 (1974) 181.

[10] PHOJET version $1.05 \mathrm{c}$ is used with default options:

R. Engel, Z. Phys. C 66 (1995) 203;

R. Engel, J. Ranft, Phys. Rev. D 54 (1996) 4246.

[11] KK2f version 4.12 is used: S. Jadach, B.F.L. Ward, Z. Wạs, Comput. Phys. Commun. 130 (2000) 260.

[12] KORALZ version 4.04 is used: S. Jadach, B.F.L. Ward, Z. Wạs, Comput. Phys. Commun. 79 (1994) 503.

[13] KORALW version 1.33 is used: M. Skrzypek, et al., Comput. Phys. Commun. 94 (1996) 216.

[14] DIAG36 Monte Carlo: F.A. Berends, P.H. Daverfeldt, R. Kleiss, Nucl. Phys. B 253 (1985) 441.

[15] GEANT version 3.15 is used: R. Brun et al., preprint CERN DD/EE/84-1 (1984), revised 1987.

[16] H. Fesefeldt, RWTH Aachen report PYTHA 85/2 (1985).

[17] P. Béné, et al., Nucl. Instrum. Methods A 306 (1991) 150; D. Haas, et al., Nucl. Instrum. Methods A 420 (1999) 101.

[18] L3 Collaboration, M. Acciarri, et al., Phys. Lett. B 519 (2001) 33.

[19] S. Catani, et al., Nucl. Phys. B 406 (1993) 187; S.D. Ellis, D.E. Soper, Phys. Rev. D 48 (1993) 3160; M. Seymour, http://hepwww.rl.ac.uk/theory/seymour/ktclus/.

[20] G. D’Agostini, Nucl. Instrum. Methods A 362 (1995) 487.

[21] R. Bizzarri, et al., Nucl. Instrum. Methods A 283 (1989) 799.

[22] S. Frixione, et al., Phys. Lett. B 319 (1993) 339.

[23] M. Gluck, E. Reya, A. Vogt, Phys. Rev. D 45 (1992) 3986; M. Gluck, E. Reya, A. Vogt, Phys. Rev. D 46 (1992) 1973.

[24] T. Kleinwort, G. Kramer, Nucl. Phys. B 477 (1996) 3; M. Klasen, T. Kleinwort, G. Kramer, Eur. Phys. J. C 1 (1998) 1.

[25] OPAL Collaboration, G. Abbiendi, et al., Eur. Phys. J. C 31 (2003) 307. 